\title{
PSYCHOLOGY
}

\section{ПСИХОАНАЛИТИЧЕСКИЙ ТРЕНИНГ КАК ИНСТРУМЕНТ РАЗВИТИЯ ПСИХОЛОГИЧЕСКИХ ГРАНИЦ ПОДРОСТКА}

\author{
Д.психол.н., профессор кафедры психологии ИПК ГСЗУ Дорожкин В. Р., \\ аспирант кафедры психологии ИПК ГСЗУ Савченко Е. В.
}

Институт подготовки кадров государственной службы занятости Украины (ИПК ГСЗУ), Украина, Киев

DOI: https://doi.org/10.31435/rsglobal_ws/31102019/6730

\section{ARTICLE INFO}

Received: 20 August 2019

Accepted: 12 October 2019

Published: 31 October 2019

\section{KEYWORDS}

psychoanalytic training, psychological boundaries of an adolescent's personality, development of psychological boundaries.

\begin{abstract}
The article is devoted to psychoanalytic training as an instrument for the development of psychological boundaries of adolescents. The base principles of the training are described and the features of its structure and duration are characterized. The topics that were put forward as the main ones for certain meetings of teenage groups are justified in detail. Psychoanalytic training has been studied in terms of its effectiveness and impact on the development of the boundaries as well as their structure and functions in adolescents. The analysis is conducted of all those mental changes and new growths due to which the formation of the boundaries of the personality takes place.
\end{abstract}

Citation: Дорожкин В. Р., Савченко Е. В. (2019) Psihoanaliticheskij Trening kak Instrument Razvitiya Psihologicheskih Granic Podrostka. World Science. 10(50), Vol.2. doi: 10.31435/rsglobal_ws/31102019/6730

Copyright: (C) 2019 Дорожкин В. Р., Савченко Е. В. This is an open-access article distributed under the terms of the Creative Commons Attribution License (CC BY). The use, distribution or reproduction in other forums is permitted, provided the original author(s) or licensor are credited and that the original publication in this journal is cited, in accordance with accepted academic practice. No use, distribution or reproduction is permitted which does not comply with these terms.

Введение. Психоаналитический тренинг - понятие, которое входит в психологическую практику только сейчас. Его предшественник - тренинговый анализ - традиционная форма подготовки начинающих психоаналитиков в рамках обучающих программ различных аналитических Институтов. К сегодняшнему дню формализованы методические рекомендации по проведению и прохождению тренингового анализа, определены и систематизированы тренинговые стандарты и процедуры, четко регламентированы условия и длительность тренингового анализа [8].

Наше понимание психоаналитического тренинга (ПТ) в том контексте, в котором мы используем его в данной работе, а именно, тренинга для подростков, отличается от традиционного подхода к тренинговому анализу (ТА). Во-первых, ПТ не несет обязательной дидактической нагрузки и не ставит перед собой целей развивать аналитическую идентичность. Во-вторых, ПТ может проводиться для всех заинтересованных в психоаналитическом подходе лиц (в частности, для подростков), а не только для слушателей психоаналитических Программ. В-третьих, длительность ПТ - 100 часов. Это в три раза меньше, чем время, которое требует традиционный TA. В-четвертых, ПТ проводится в рамках двухдневных аналитических групп (по 20 часов каждая), что также отличает его от ТА, который проходит по часу несколько раз в неделю. В-пятых, участие в ПТ исключительно добровольное занятие. Тем не менее, мы заключали устный контракт с подростками и их родителями о том, что, если они принимают решение участвовать в нашем исследовании, то обязуются быть на пяти групповых встречах (по 20 часов каждая), а также в перерывах между встречами пройти 30 часов индивидуальной психоаналитической терапии.

Наконец, самое главное, в нашем понимании ПТ - это специального рода инструмент по развитию психологических границ подростков. 
В качестве цели данной работы мы решили исследовать, как можно развивать психологические границы подростков с помощью ПТ. Психоаналитический тренинг открывает новые средства понимания как самих психологических границ подростков, так и психологических инструментов их формирования.

Ключевая идея нашей статьи состоит в том, что в подростковом возрасте формирование границ и пересмотр механизмов защиты происходят стихийно. Поэтому мы решили исследовать, как влияет психоаналитический тренинг на развитие психологических границ и возможно ли с помощью специальной психологической работы это развитие упорядочить.

Результаты исследований. Мы провели отбор из 83 подростков, в результате которого было отобрано 46 участников. В дальнейшем мы сформировали из них четыре группы по 10-12 человек. Также было получено разрешение родителей на прохождение их детьми ПТ.

Программа ПТ была адаптирована для подростковой аудитории. При этом мы исходили из ряда условий:

1. Программа, упражнения и интерпретации коммуникаций между участниками должны базироваться на психоаналитических принципах и методах работы.

2. В программе допускалось использование определенных тренинговых игр, упражнений и арт-терапевтических процедур, но обратная связь, а также обсуждение результатов должны были структурироваться в рамках аналитического понимания природы психики и групповых явлений.

3. Значительное внимание в ПТ было уделено работе с психологическими защитами, которые опосредовали взаимоотношения участников между собой, их переносным реакциям, а также выстраиваемым ими коммуникациям с аналитиком и группой.

4. Акцент в ПТ делался на внутренних и внешних психологических границах участников. Подростки исследовали свои и чужие границы, переживания и фантазии, с ними связанные.

5. Конечной целью ПТ было формирование навыков управления собственными психологическими границами, а также осознание тех психологических защит, которые использовались участниками в тот или иной момент времени и опосредовали работу внутренних и внешних психологических границ.

В связи с тем, что центральным предметом исследования ПТ являлись внешние психологические границы и внутренние Я-отграничения (термин Я-отграничения ввел Г. Аммон [7]), а также системы обслуживающих их защитных механизмов, мы подробно изучали их трансформацию в процессе работы группы. Делали это опять же с помощью средств, которые предоставляет психоанализ. Для этого мы отслеживали несколько пластов интрасубъективных и интерсубъективных отношений:

1. Наблюдали избирательность контактов в группе, выбор партнеров по общению, формирование сети межличностных предпочтений и антипатий, особенности формирования матрицы переносных реакций участников. Этот пласт коммуникации базируется на поддержании баланса между личностной автономией и стремлением принадлежать к той или иной микрогруппе. Для нас было существенным, что эти отношения регулируются внешними психологическими границами и системами механизмов защиты участников.

2. Изучали формирование и проявления чувства «потери целостности Я» и диффузии идентичности [11], которые возникали вследствие сближения и периодического слияния участников группы. Подобные сближения являются закономерными в групповом процессе. Они связаны с ложными распознаваниями других, как самих себя, потребностями определить анаклетический объект в группе, желаниями присвоить терапевта или быть присвоенными им. Мы исследовали процессы воспроизводства Я и отмежевания от не-Я, и анализировали, как эти процессы влияют на дифференциацию психического пространства подростков.

3. Изучали реакцию участников группы на подростковое «чувство взрослости». В процессе работы нашей группы подростки совершали колебательные движения из позиции ребенка в позицию взрослого множество раз. В частности, принималась и отвергалась сексуальность и связанное с ней восприятие подростка как сексапильного Объекта; присваивалась и отщеплялась ответственность за свое поведения, собственные чувства и за жизнь в целом и пр. Интересно, что подросткам так же трудно было принять взрослость других участников, как и собственную. Зачастую, они пользовались проекцией и смещали неуверенность в собственном взрослом статусе на других. Эти процессы иллюстрировали работу Я-отграничений в тех их функциях, которые связаны с отделением Я от не-Я (отграничивающая функция), с удержанием отдельных чувств и регулированием обмена содержанием между Я и социальным окружением (фильтрующая функция), с динамическим изменением границ Я путем их расширения (развитийная функция психологических границ). 
4. Анализировали реанимацию триангулярных объектных отношений (эдипов комплекс), которые происходили в жизни подростков в их актуальном времени и дополнительно были усилены переносными взаимоотношениями с психоаналитиком [9]. В процессе анализа групповых ассоциаций, фантазий и переносных реакций мы установили, что пересмотр объектов влечения влечет изменения содержания Сверх-Я и подвергает ревизии Яотграничения между Я и Сверх-Я. Последние начинают лучше регулировать обмен между Я и Сверх-Я (фильтрующая функция), корректнее дифференцируют инстанции психики (отграничивающая функция). Данные процессы поддерживаются также канализирующей и стабилизирующее-интегрирующей функциями психологических границ.

5. Отслеживали развитие самосознания подростков в той его части, которая касается проявлений их индивидуального Я. Индивидуализация поведения и ответственных за него частей личности обеспечиваются формированием психологических границ в отграничивающей, сдерживающей, фильтрующей, стабилизирующе-интегрирующей, развитийной и дифференцирующей функциях. Развитие самосознания подростков также связано с изменениями конституционных, динамических и структурных свойств границ. В частности, Я-отграничения между Я и Сверх-Я становятся более проницаемыми, за счет чего развивается морально-этическая сторона личности. Наоборот, Я-отграничения между Я и Оно уплотняются, что влечет сдерживание бессознательных импульсов и нарастающего сексуального желания. При этом личностные границы подростка расширяются, твердеют и, зачастую, агрессивно отстаиваются.

Выше по тексту было сказано, что программа ПТ состояла из пяти двухдневных групп и 30 индивидуальных сессий между ними. Структура каждой двухдневной аналитической группы была идентична.

День 1.

Модуль 1. Групповая обратная связь. Прояснение запросов. Групповое обсуждение

Модуль 2. Просмотр фильма. Обсуждение.

Модуль 3. Групповая аналитическая терапия.

Модуль 4. Групповая арт-терапия. Обсуждение. Обратная связь.

День 2.

Модуль 5. Теория по теме.

Модуль 6. Групповая аналитическая терапия.

Модуль 7. Просмотр фильма. Обсуждение.

Модуль 8. Групповая аналитическая терапия. Обсуждение. Обратная связь.

Каждая двухдневная аналитическая группа была тематической. В подборе тем мы руководствовались нашим исследованием, но делали эти темы понятными и доступными для самих подростков. Таким образом, аналитические двухдневные встречи получили названия:

Первая группа. Матрица отношений и психологические границы личности. Свойства психологических границ.

Вторая группа. Формирование Я и «не-Я» в подростковом возрасте. Внутренние и внешние Я-отграничения. Функции Я-отграничений.

Третья группа. Подростковое чувство взрослости: на пути к сепарации. Тревога и защитные механизмы.

Четвертая группа. Особенности выбора объектов любви в возрасте от 14 до 16 лет. Взаимоотношения с родителями сквозь призму Эдипова комплекса.

Пятая группа. Что делает меня уникальным? Осознание особенностей собственной личности. Как отличить истинное Я от проекта родителей

В подборе тем мы сделали акцент на основных психических новообразованиях подросткового возраста. Кроме этого, мы учли те важности, которые связаны с коммуникацией в пространстве межличностных и внутриличностных отношений и проявляются в пубертате. Вне зависимости от основной темы аналитической группы, каждый раз мы отслеживали изменения внутренних и внешних психологических границ. Дополнительно к этому, мы изучали работу механизмов психологических защит ребят, наблюдали трансформацию этой работы по мере прохождения ПТ.

Анализ и интерпретацию результатов ПТ мы осуществляли в двух плоскостях:

1. Статистически исследовали количественные показатели произошедших изменений психологических границ у подростков. 
2. Планомерно с помощью психоаналитических средств изучали качественные сдвиги и трансформации, произошедшие у наших участников в процессе психоаналитических сессий и двухдневных групп.

Мы протестировали ребят во всех четырех группах до и после ПТ. В эмпирической части работы приняло участие 46 подростков. Сами этапы тестирования проходили с разницей в 10 месяцев. Диагностика осуществлена с помощью двух методик: «Психологические границы» (Т.С. Леви) [5] и «Я-структурный тест Г. Аммона» (ISTA) [7]. В связи с тем, что основную часть изменений подростки фиксировали и обсуждали в процессе психотерапевтической работы на группах, указанных двух методик было достаточно.

Для выявления различий между результатами двухкратного тестирования одной выборки испытуемых с помощью методики «Психологические границы» (Т.С. Леви), мы воспользовались Т-критерием Вилкоксона для зависимых групп данных. Результаты представлены в Таблицах 1-6. По результатам можно сделать следующие выводы:

1. Т-критерий Вилкоксона для зависимых групп данных не выявил различий между двумя результатами тестирований (до и после проведенного ПТ) по таким функциям границ: активно невпускающая функция $\left(\mathrm{T}_{\mathrm{e}}=358,5\right.$ при $\mathrm{p}=0,245$, Таблица 1$)$, активно вбирающая функция $\left(\mathrm{T}_{\mathrm{e}}=\right.$ 336,5 при $\mathrm{p}=0,15$, Таблица 3) и отдающая функция $\left(\mathrm{T}_{\mathrm{e}}=396\right.$ при $\mathrm{p}=0,353$, Таблица 4).

2. Т-критерий Вилкоксона позволил статистически достоверно подтвердить рост показателей после проведенного ПТ по следующим свойствам границ: активно сдерживающая функция $\left(\mathrm{T}_{\mathrm{e}}=253\right.$ при $\mathrm{p}=0,002 \leq \mathrm{p}=0,01$, Таблица 5) и спокойно-нейтральная функция $\left(\mathrm{T}_{\mathrm{e}}=\right.$ 145 при $\mathrm{p}=0,0006 \leq \mathrm{p}=0,001$, Таблица 6).

3. Т-критерий установил статистически значимое уменьшение значений по проницаемой функции психологических границ у подростков $\left(\mathrm{T}_{\mathrm{e}}=249\right.$ при $\mathrm{p}=0,0011 \leq \mathrm{p}=0,01$, Таблица 2)

Обнаруженные статистические различия свидетельствуют в пользу влияния ПТ на возможности участников контейнировать внутренние нужды, потребности, желания и в целом психическую энергию. ПТ позволил подросткам сформировать дополнительные умения сдерживать внутренние импульсы, что свидетельствует не только в пользу развития волевых качеств, но также отражает приобретенные ребятами способности к осознанию и удержанию локализованными различных фрагментов психического опыта и прикрывающих их защитных механизмов.

Достоверное увеличение значений по спокойно-нейтральной функции в результате ПТ указывает на развитие у подростков навыков поддержания внутреннего и внешнего балансов в периоды развития гармоничного поля отношений. Данное психическое новоприобретение указывает на формирование чувств «внутренней безопасности» и «спокойного отношения к миру». Подобные состояния усиливают у подростков переживания чувства взрослости, что дополнительно свидетельствует в пользу зрелости личности.

Уменьшение значений по проницаемой функции психологических границ, с одной стороны, влечет большую автономию и «фильтрацию» материала, продуцируемого внешними Объектами, а с другой, обеспечивает процессы сепарации необходимыми инструментами. Развитие в сторону закрытости и герметичности личности позволяет подросткам в достаточной степени обособиться от родительской семьи и открыться новым партнерским отношения.

Таблица 1. Отличия между группами данных до и после прохождения ПТ по шкале «активно невпускающая функция» границы по методике «Психологические границы» (Т.С.Леви) (Т-критерий Вилкоксона)

\begin{tabular}{|l|l|r|r|r|}
\hline \multirow{2}{*}{ Pair of Variables } & \multicolumn{4}{|l|}{$\begin{array}{l}\text { Wilcoxon Matched Pairs Test (Spreadsheet_new) } \\
\text { Marked tests are significant at } \mathrm{p}<, 05000\end{array}$} \\
\cline { 2 - 6 } & $\begin{array}{c}\text { Valid } \\
\mathrm{N}\end{array}$ & $\mathrm{T}$ & $\mathrm{Z}$ & $\mathrm{p}$-value \\
\hline АНВГран \& АНВГранПТ & 42 & $358,500 \mathrm{C}$ & 1,162841 & 0,244895 \\
\hline
\end{tabular}

АНВГран - это активно невпускающая функция до прохождения ПТ

АНВГранПТ - активно невпускающая функция после прохождения ПТ 
Таблица 2. Отличия между группами данных до и после прохождения ПТ по шкале «проницаемая функция» границы по методике «Психологические границы» (Т.С.Леви) (Т-критерий Вилкоксона)

\begin{tabular}{|c|c|c|c|c|}
\hline \multirow[b]{2}{*}{ Pair of Variables } & \multicolumn{4}{|c|}{$\begin{array}{l}\text { Wilcoxon Matched Pairs Test (Spreadsheet_new) } \\
\text { Marked tests are significant at } p<, 05000\end{array}$} \\
\hline & $\begin{array}{l}\text { Valid } \\
\mathrm{N}\end{array}$ & $\mathrm{T}$ & Z & $\mathrm{p}$-value \\
\hline ПронГран \& ПронГранПТ & 42 & $249,000 \mathrm{C}$ & 2,531992 & 0,011342 \\
\hline
\end{tabular}

ПронГран - это проницаемая функция границы до прохождения ПТ

ПронГранПТ - проницаемая функция границы после прохождения ПТ

Таблица 3. Отличия между группами данных до и после прохождения ПТ по шкале «активно вбирающая функция» границы по методике «Психологические границы» (Т.С.Леви) (Т-критерий Вилкоксона)

Wilcoxon Matched Pairs Test (Spreadsheet_new)

Marked tests are significant at $p<, 05000$

Pair of Variables

\begin{tabular}{|c|c|c|c|} 
Valid & $T$ & $\mathrm{Z}$ & $\mathrm{p}$-value
\end{tabular}

$\mathrm{N}$

\begin{tabular}{lrr}
42 & $336,500 \mathrm{C}$ & 1,437921 \\
\hline
\end{tabular}

0,150457

АВбирГран - активно вбирающая функция границы до прохождения ПТ

АВбирГранПТ - активно вбирающая функция границы после прохождения ПТ

Таблица 4. Отличия между группами данных до и после прохождения ПТ по шкале «отдающая функция» границы по методике «Психологические границы» (Т.С.Леви) (Т-критерий Вилкоксона)

\begin{tabular}{|c|c|c|c|c|}
\hline \multirow[b]{2}{*}{ Pair of Variables } & \multicolumn{4}{|c|}{$\begin{array}{l}\text { Wilcoxon Matched Pairs Test (Spreadsheet_new } \\
\text { Marked tests are significant at } p<, 05000\end{array}$} \\
\hline & $\begin{array}{c}\text { Valid } \\
\mathrm{N}\end{array}$ & $\mathrm{T}$ & Z & $\mathrm{p}$-value \\
\hline АОтдГран \& АОтдГранПТ & 43 & $396,000 \mathrm{C}$ & 0,929771 & $0,35249 \mathrm{C}$ \\
\hline
\end{tabular}

АОтдГран - активно отдающая функция границы до прохождения ПТ

АОтдГранПТ - активно отдающая функция границы после прохождения ПТ

Таблица 5. Отличия между группами данных до и после прохождения ПТ по шкале «активно сдерживающая функция» границы по методике «Психологические границы» (Т.С.Леви) (Т-критерий Вилкоксона)

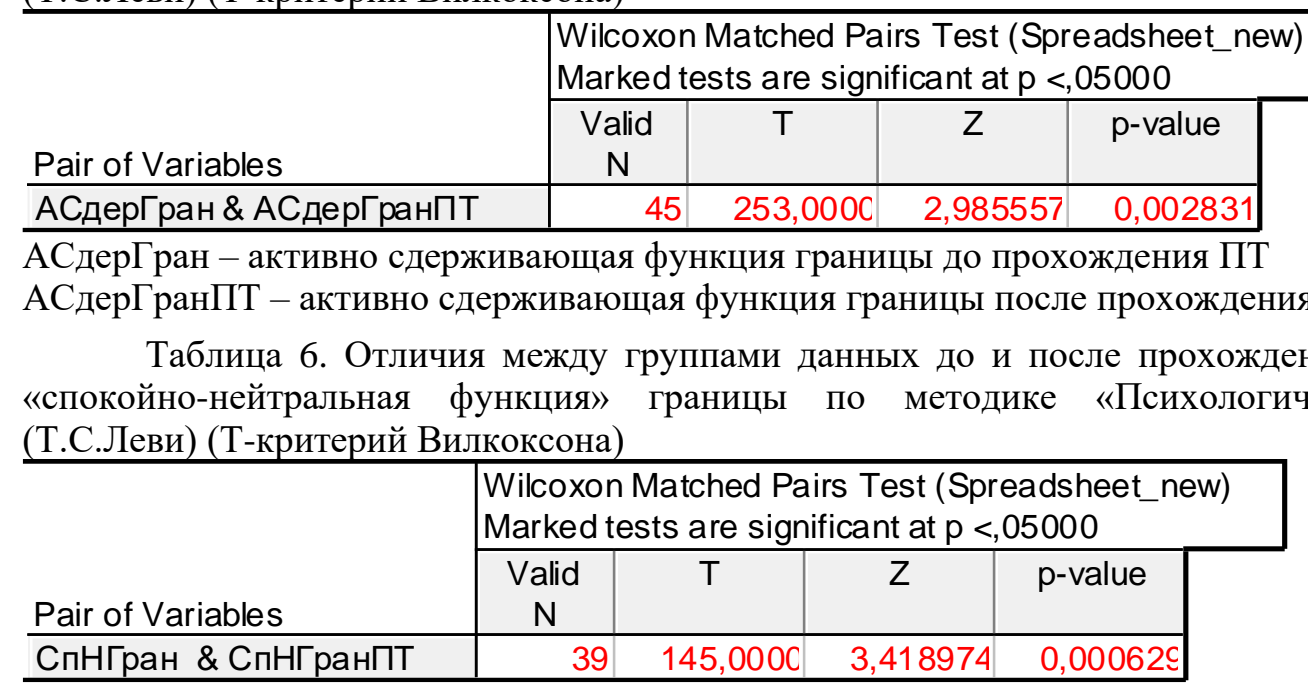

СпНГран - спокойно-нейтральная функция границы до прохождения ПТ

СпНГранПТ - спокойно-нейтральная функция границы после прохождения ПТ

Качественный анализ произошедших трансформаций по параметрам методики «Психологические границы» позволяет нам утверждать не столько изменения свойств границ, сколько их осознанное развитие. В обратной связи после каждой из психотерапевтических 
групп подростки указывали на обнаруженные ими умения в управлении границами и обслуживающими их защитными механизмами.

Различия во внешних и внутренних структурах границ личности мы устанавливали с помощью Т-критерия Вилкоксона для зависимых групп данных. Т-критерий был применен к результатам двукратного тестирования нашей выборки из 46 подростков с помощью «Я-структурного теста Аммона» (ISTA). Результаты представлены в Таблицах 7-14.

По материалам этих таблиц мы можем сделать следующие выводы:

1. Статистически достоверно выросли показатели по таким структурным свойствам внутренних и внешних Я-отграничений:

а. После ПТ увеличился параметр внешнее конструктивное Я-отграничение $\left(\mathrm{T}_{\mathrm{e}}=93\right.$ при $\mathrm{p}=0,000012 \leq \mathrm{p}=0,0001$, Таблица 7).

b. Значительно изменилось в сторону увеличения внутреннее конструктивное Я-отграничение $\left(\mathrm{T}_{\mathrm{e}}=88\right.$ при $\mathrm{p}=0,00099 \leq \mathrm{p}=0,001$, Таблица 11$)$.

2. Достоверно снизились показатели по следующим структурным свойствам Я-отграничений:

а. Статистически достоверно уменьшился параметр внешнее дефицитарное Я-отграничение $\left(\mathrm{T}_{\mathrm{e}}=20\right.$ при $\mathrm{p}=0,000001 \leq \mathrm{p}=0,0001$, Таблица 9).

b. После ПТ снизился показатель по внутреннему деструктивному Я-отграничению $\left(\mathrm{T}_{\mathrm{e}}=160\right.$ при $\mathrm{p}=0,03 \leq \mathrm{p}=0,05$, Таблица 12).

с. Значительно меньше стали значения и по внутреннему дефицитарному Я-отграничению ( $\mathrm{T}_{\mathrm{e}}=0$ при $\mathrm{p}=0,00000 \leq \mathrm{p}=0,0001$, Таблица 13).

d. Достоверно снизились показатели по внутреннему общему Я-отграничению $\left(\mathrm{T}_{\mathrm{e}}=30,5\right.$ при $\mathrm{p}=0,00000 \leq \mathrm{p}=0,0001$, Таблица 14).

3. Такие показатели структурных свойств границ, как: внешнее деструктивное Я-отграничение и внешнее общее Я-отграничение не поменялись или изменились статистически недостоверно.

Качественный анализ статистических данных позволяет говорить о значительном преобразовании структуры внутренних и внешних Я-отграничений. Трансформации и развитие границ особенно коснулись внутренних Я-отграничений. Конструктивные внутренние Я-отграничения получили развитие в плане роста их показателей. Наоборот, деструктивные и дефицитарные внутренние Я-отграничения оказались значительно сниженными по результатам повторного тестирования и обработки данных с помощью Т-критерия Вилкоксона для зависимых групп данных.

Выводы. Выявленные статистические закономерности позволяют говорить об эффективности проведенной программы ПТ. Психоаналитический тренинг позволил не только развить психологические границы по функциям и структурным свойствам, но и способствовал качественной перестройке работы границ.

Участники ПТ неоднократно комментировали те изменения, которые происходили у них самих или других членов группы. Они отслеживали работу механизмов защиты и то, как последние опосредовали установление границ в группе. Дополнительно к сказанному, ребята приобрели навыки анализа интрасубъективного пространства по интерсубъективным коммуникациям. Это позволило делать комментарии по трансформации внутренних Я-отграничений других участников и по тому, как эти трансформации влияют на устанавливаемые отношения в группе.

Таким образом, развитие, которое получили психологические границы и системы защитных механизмов в ПТ, отражает значительный уровень трансформации личности подростка, при которой его психика приобретает более высокую внутреннюю дифференциацию. Последнее помогает выстраивать и управлять коммуникативными процессами между внутренними частями Я и внешним миром, а также между отдельными аспектами внутренних содержаний Я и не-Я. Такие изменения создают предпосылки для развития более гармоничной личности подростков с устойчивым самоотношением. Собственно, об этом неоднократно свидетельствовали сами участники наших групп на заключительных стадиях ПТ.

Тот факт, что, несмотря на ПТ и создание условий к изменениям, не все показатели Я-отграничений поменялись, лишний раз свидетельствует о том, что развитие границ происходит гетерогенно и имеет сложную динамику. 
Таблица 7. Отличия между группами данных до и после прохождения ПТ по шкале «внешнее конструктивное Я-отграничение» методики «Я-структурный тест Г.Амона» (ISTA) (Т-критерий Вилкоксона)

\begin{tabular}{|c|c|c|c|c|}
\hline & \multicolumn{4}{|c|}{$\begin{array}{l}\text { Wilcoxon Matched Pairs Test (Spreadsheet_new } \\
\text { Marked tests are significant at } p<, 05000\end{array}$} \\
\hline & $\begin{array}{c}\text { Valid } \\
\mathrm{N}\end{array}$ & $\mathrm{T}$ & Z & $\mathrm{p}$-value \\
\hline
\end{tabular}

ВнЯОК - внешнее конструктивное Я-отграничение до прохождения ПТ

ВнЯОКПТ - внешнее конструктивное Я-отграничение после прохождения ПТ

Таблица 8. Отличия между группами данных до и после прохождения ПТ по шкале «внешнее деструктивное Я-отграничение» методики «Я-структурный тест Г.Амона» (ISTA) (Т-критерий Вилкоксона)

\begin{tabular}{|l|l|c|c|c|c|}
\hline \multirow{3}{*}{ Pair of Variables } & \multicolumn{5}{|l|}{ Wilcoxon Matched Pairs Test (Spreadsheet_new) } \\
& Marked tests are significant at $\mathrm{p}<, 05000$ \\
\cline { 2 - 5 } & $\begin{array}{c}\text { Valid } \\
\mathrm{N}\end{array}$ & $\mathrm{T}$ & $\mathrm{Z}$ & $\mathrm{p}$-value & \\
\hline ВнЯОДес \& ВнЯОДесПТ & 37 & $350,000 \mathrm{C}$ & $0,02262 \mathrm{C}$ & 0,981946 \\
\hline
\end{tabular}

ВнЯОДес - внешнее деструктивное Я-отграничение до прохождения ПТ

ВнЯОДесПТ - внешнее деструктивное Я-отграничение после прохождения ПТ

Таблица 9. Отличия между группами данных до и после прохождения ПТ по шкале «внешнее дефицитарное Я-отграничение» методики «Я-структурный тест Г.Амона» (ISTA) (Т-критерий Вилкоксона)

\begin{tabular}{|c|c|c|c|c|}
\hline \multirow[b]{2}{*}{ Pair of Variables } & \multicolumn{4}{|c|}{$\begin{array}{l}\text { Wilcoxon Matched Pairs Test (Spreadsheet_new) } \\
\text { Marked tests are significant at } p<, 05000\end{array}$} \\
\hline & $\begin{array}{c}\text { Valid } \\
\mathrm{N}\end{array}$ & $\bar{T}$ & $\mathrm{Z}$ & $p$-value \\
\hline ВнЯОДеф \& ВнЯОДефПТ & 37 & $20,0000 \mathrm{C}$ & 5,001104 & 0,000001 \\
\hline
\end{tabular}

ВнЯОДеф - внешнее дефицитарное Я-отграничение до прохождения ПТ

ВнЯОДефПТ - внешнее дефицитарное Я-отграничение после прохождения ПТ

Таблица 10. Отличия между группами данных до и после прохождения ПТ по шкале «внешнее общее Я-отграничение» методики «Я-структурный тест Г.Амона» (ISTA) (Т-критерий Вилкоксона)

\begin{tabular}{|c|c|c|c|c|}
\hline \multirow[b]{2}{*}{ Pair of Variables } & \multicolumn{4}{|c|}{$\begin{array}{l}\text { Wilcoxon Matched Pairs Test (Spreadsheet_new) } \\
\text { Marked tests are significant at } p<, 05000\end{array}$} \\
\hline & $\begin{array}{l}\text { Valid } \\
\mathrm{N}\end{array}$ & $\mathrm{T}$ & Z & $p$-value \\
\hline ВнЯООб \& ВнЯООбПТ & 34 & $259,500 \mathrm{C}$ & $0,64966 \varepsilon$ & 0,515907 \\
\hline
\end{tabular}

ВнЯООб - внешнее общее Я-отграничение до прохождения ПТ

ВнЯООбПТ - внешнее общее Я-отграничение после прохождения ПТ

Таблица 11. Отличия между группами данных до и после прохождения ПТ по шкале «внутреннее конструктивное Я-отграничение» методики «Я-структурный тест Г.Амона» (ISTA) (Т-критерий Вилкоксона)

\begin{tabular}{|c|c|c|c|c|}
\hline \multirow[b]{2}{*}{ Pair of Variables } & \multicolumn{4}{|c|}{$\begin{array}{l}\text { Wilcoxon Matched Pairs Test (Spreadsheet_new } \\
\text { Marked tests are significant at } p<, 05000\end{array}$} \\
\hline & $\begin{array}{c}\text { Valid } \\
\mathrm{N}\end{array}$ & $\mathrm{T}$ & Z & $p$-value \\
\hline ВнутЯОК \& ВнутЯОКПТ & 32 & 88,00000 & 3,291013 & 0,000998 \\
\hline
\end{tabular}

ВнутЯОК - внутреннее конструктивное Я-отграничение до прохождения ПТ

ВнутЯОКПТ - внутреннее конструктивное Я-отграничение после прохождения ПТ 
Таблица 12. Отличия между группами данных до и после прохождения ПТ по шкале «внутреннее деструктивное Я-отграничение» методики «Я-структурный тест Г.Амона» (ISTA) (Т-критерий Вилкоксона)

\begin{tabular}{|c|c|c|c|c|}
\hline \multirow[b]{2}{*}{ Pair } & \multicolumn{4}{|c|}{$\begin{array}{l}\text { Wilcoxon Matched Pairs Test (Spreadsheet_new) } \\
\text { Marked tests are significant at } p<, 05000\end{array}$} \\
\hline & $\begin{array}{c}\text { Valid } \\
\mathrm{N}\end{array}$ & $\mathrm{T}$ & Z & $p$-value \\
\hline
\end{tabular}

ВнутЯОДес - внутреннее деструктивное Я-отграничение до прохождения ПТ

ВнутЯОДесПТ - внутреннее деструктивное Я-отграничение после прохождения ПТ

Таблица 13. Отличия между группами данных до и после прохождения ПТ по шкале «внутреннее дефицитарное Я-отграничение» методики «Я-структурный тест Г.Амона» (ISTA) (Т-критерий Вилкоксона)

\begin{tabular}{|c|c|c|c|c|}
\hline \multirow[b]{2}{*}{ Pair of Variables } & \multicolumn{4}{|c|}{$\begin{array}{l}\text { Wilcoxon Matched Pairs Test (Spreadsheet_new) } \\
\text { Marked tests are significant at } \mathrm{p}<, 05000\end{array}$} \\
\hline & $\begin{array}{c}\text { Valid } \\
\mathrm{N}\end{array}$ & $\mathrm{T}$ & Z & p-value \\
\hline ВнутЯОДеф \& ВнутЯОДефрПТ & 45 & 0,00 & 5,841308 & 0,000000 \\
\hline
\end{tabular}

ВнутЯОДеф - внутреннее дефицитарное Я-отграничение до прохождения ПТ

ВнутЯОДефПТ - внутреннее дефицитарное Я-отграничение после прохождения ПТ

Таблица 14. Отличия между группами данных до и после прохождения ПТ по шкале «внутреннее общее Я-отграничение» методики «Я-структурный тест Г.Амона» (ISTA) (Т-критерий Вилкоксона)

\begin{tabular}{|c|c|c|c|c|}
\hline \multirow[b]{2}{*}{ Pair of Variables } & \multicolumn{4}{|c|}{$\begin{array}{l}\text { Wilcoxon Matched Pairs Test (Spreadsheet_nen } \\
\text { Marked tests are significant at } p<, 05000\end{array}$} \\
\hline & $\begin{array}{c}\text { Valid } \\
\mathrm{N}\end{array}$ & $\bar{T}$ & Z & $\mathrm{p}$-value \\
\hline ВнутЯООб \& ВнутЯООбПТ & 45 & $30,5000 \mathrm{C}$ & $5,49703 \varepsilon$ & $0,00000 C$ \\
\hline
\end{tabular}

ВнутЯООб - внутреннее общее Я-отграничение до прохождения ПТ

ВнутЯООбПТ - внутреннее общее Я-отграничение после прохождения ПТ

\section{REFERENCES}

1. Vinnikot D. V. Malen'kie deti i ikh materi / D. V. Vinnikot - Moscow: Klass, 1998. - 80 p.

2. Kernberg O.F. Vazhki osobystisni rozlady. Strategii' psyhoterapii' / Per. z angl. M.I. Zavalova. - Moscow: Nezalezhna firma «Klas», 2000. - $464 \mathrm{p}$.

3. Kljajn M. Zazdrist' i podjaka / M. Kljajn. - Saint-Petersburg: B.S.K., 1997. - 96 p.

4. Kokhut Kh. Analiz samosti: Sistematicheskii podkhod k lecheniiu nartsissicheskikh narushenii lichnosti / Khaints Kokhut. - Moscow: «Kogito-Tsentr», 2003. - 368 p.

5. Levi T.S. Metodika diagnostiki psikhologicheskoi granitsy lichnosti / T.S. Levi // Voprosy psikhologii. 2013 - № 1. - pp. 131-146.

6. Nartova-Bochaver S. K. Poniatie «psikhologicheskoe prostranstvo lichnosti»: obosnovanie i prikladnoe znachenie / S.K. Nartova-Bochaver // Psikhologicheskii zhurnal. - 2003. - T. 24. - № 6. - pp. $27-36$.

7. Ocherki dinamicheskoi psikhiatrii. Transkul'tural'noe issle-dovanie / Pod. red. M.M. Kabanova, N.G. Neznanova. - Saint-Petersburg: Institut im. V.M. Bekhtereva, 2003. - 438 p.

8. Fai M. Put' analitika. Osnovnye printsipy psikhoanaliticheskoi tekhniki: per. s angl. / M. Fai // Pod nauch. red. prof. M. M. Reshetnikova. -SPb.: Vostochno-Evropeiskii institut psikhoanaliza, 2011. - pp. 102-108.

9. Freud S. Three essays on the theory of sexuality / S. Freud // Standart Edition. London: Hogarth Press, 1953. - pp. 135-243.

10. Shamshikova E.O. Osobennosti vzaimosviazi tipov psikhologiche-skikh granits i zashchitnykh mekhanizmov lichnosti v starshem podrostko-vom vozraste (mezhnatsional'nye razlichiia) / E.O. Shamshikova // Si-birskii pedagogicheskii zhurnal. - 2014. - №6. - pp. 178-185.

11. Erikson E. Childhood and society / E. Erikson. - New York: Norton, 1950. - 592 p. 